\section{DETERMINATION OF BOUNDS FOR THE SOLUTIONS TO THOSE BINARY DIOPHANTINE EQUATIONS THAT SATISFY THE HYPOTHESES OF RUNGE'S THEOREM*}

\author{
BY
}

DAVID LEE HILLIKER AND E. G. STRAUS ${ }^{1}$

\begin{abstract}
In 1887 Runge [13] proved that a binary Diophantine equation $F(x, y)$ $=0$, with $F$ irreducible, in a class including those in which the leading form of $F$ is not a constant multiple of a power of an irreducible polynomial, has only a finite number of solutions. It follows from Runge's method of proof that there exists a computable upper bound for the absolute value of each of the integer solutions $x$ and $y$. Runge did not give such a computation. Here we first deduce Runge's Theorem from a more general theorem on Puiseux series that may be of interest in its own right. Second, we extend the Puiseux series theorem and deduce from the generalized version a generalized form of Runge's Theorem in which the solutions $x$ and $y$ of the polynomial equation $F(x, y)=0$ are integers, satisfying certain conditions, of an arbitrary algebraic number field. Third, we compute bounds for the solutions $(x, y) \in \mathbf{Z}^{2}$ in terms of the height of $F$ and the degrees in $x$ and $y$ of $F$.
\end{abstract}

1. Introduction. Runge [13] established that certain binary Diophantine equations have only finitely many solutions. We formulate his hypotheses by introducing the following definitions for a polynomial

$$
F(x, y)=\sum_{i=0}^{d_{1}} \sum_{j=0}^{d_{2}} a_{i j} x^{i} y^{j}
$$

of degree $d_{1}$ and $d_{2}$ in $x$ and $y$ respectively.

1.1. Definitions. Let $\lambda>0$. Then $F_{\lambda}(x, y)$, the $\lambda$-leading part of $F$, is the sum of all terms $a_{i j} x^{i} y^{j}$ of $F$ for which $i+\lambda j$ is maximal. The leading part of $F$, denoted by $\tilde{F}(x, y)$, is the sum of all monomials of $F$ which appear in any $F_{\lambda}$ as $\lambda$ varies.

The sum of the terms of maximal degree, which in our notation is $F_{1}$, is the leading form or leading homogeneous part of $F$.

If $F$ factors into polynomial factors $A, B$, then $F_{\lambda}=A_{\lambda} B_{\lambda}$, although in general $\tilde{F} \neq \tilde{A} \tilde{B}$.

Received by the editors October 14, 1982.

1980 Mathematics Subject Classification. Primary 10B10, 10B15; Secondary 14H05.

Key words and phrases. Algebraic function, Diophantine equation, Puiseux series.

* It is with great regret I announce that my teacher, Ph.D. thesis advisor, and friend, Ernst Gabor Straus, died on July 12,1983 as a result of a heart attack. His departure will create a void that will be felt throughout the mathematical community. It will be particularly felt by those of us who have had the unique opportunity of studying and publishing papers with Professor Straus.

' Work of the second author was supported in part by NSF Grant MCS 79-03162. 
As usual, $\mathbf{Z}$ denotes the integers, $\mathbf{Q}$ the rationals and $\mathbf{C}$ the complex numbers. The Cartesian product of a set $S$ with itself is $S^{2}$ and $S[x], S[x, y]$ denote the sets of polynomials, with coefficients in $S$, in $x$ and $x, y$, respectively.

We can now formulate Runge's hypotheses.

1.2. Definition. Let $F(x, y) \in \mathbf{Z}[x, y]$ be irreducible in $\mathbf{Z}[x, y]$. Then $F$ satisfies Runge's Condition unless there exists a $\lambda$ so that $\tilde{F}=F_{\lambda}$ is a constant multiple of a power of an irreducible polynomial in $\mathbf{Z}[x, y]$.

Runge's Theorem. If $F$ satisfies Runge's Condition, then the Diophantine equation $F(x, y)=0$ has only finitely many solutions $(x, y) \in \mathbf{Z}^{2}$.

An independent proof of Runge's Theorem was given by Skolem [17].

In $\S 2$ we reprove Runge's Theorem in a manner resembling his original proof. However, we prove that a general class of Puiseux series with sufficiently many initial algebraic coefficients represent algebraic functions whose defining equation violates Runge's Condition whenever its curve passes through infinitely many lattice points $(x, y) \in \mathbf{Z}^{2}$. We then deduce Runge's Theorem by applying this result to the Puiseux series expansion about infinity of the algebraic function $y$ defined by $F(x, y)=0$.

We next genralize the Puiseux series theorem to curves which pass through infinitely many algebraically integral lattice points, subject to suitable restrictions (see also Hilliker and Straus [6]). We then obtain a corresponding generalization of Runge's Theorem for binary Diophantine equations over an algebraic number field.

In $\S 3$ we compute bounds for the solutions $(x, y) \in \mathbf{Z}^{2}$ for some special cases of those Diophantine equations $F(x, y)=0$ that satisfy Runge's Condition. We develop a sufficient condition for all the coefficients in the Puiseux series expansion

$$
y=a_{-m} x^{m / e}+a_{-m+1} x^{(m-1) / e}+a_{-m+2} x^{(m-2) / e}+\cdots
$$

( $m, e \in \mathbf{Z}, e>0,|x|>R$ ) to be in the field of the leading coefficient $a_{-m}$, and treat this case (Theorem 3.31).

In $\S 4$ we treat the general case, which involves more computational complexity, where the Puiseux coefficients need not be in the field of the leading coefficient. We compute a bound (Theorem 4.9) valid for all Diophantine equations that satisfy Runge's Condition.

Hilliker $[3,4]$ has developed techniques for solving certain Diophantine equations. To illustrate those techniques the quartic case of Runge's Theorem has been developed (Hilliker [4]). The methods of the papers [3, 4] are different from those of the present paper, but both approaches rest upon certain numerical techniques in the classical theory of algebraic functions.

Schinzel [14] used Siegel's Theorem [16] to sharpen Runge's Theorem. His results do not lead to computable bounds, since they depend on Siegel's Diophantine approximation methods [15], and we, therefore, do not consider them here.

For more on Runge's Theorem see also Ellison [1], Maillet [8, 9], Mordell [10] and Skolem [18]. Mordell proves, but does not compute bounds for, the special case of an irreducible equation where the leading form $F_{1}$ is not a constant times a power of an irreducible polynomial. Skolem gives an informative historical discussion of the events up to 1938. 
1.3. Some algebraic concepts and notation. An algebraic number $\theta$ is the solution of an equation $P(x)=0$ where

$$
P(x)=a_{n} x^{n}+a_{n-1} x^{n-1}+\cdots+a_{0}, \quad a_{n}>0,
$$

is an irreducible polynomial in $\mathbf{Z}[x]$. The polynomial $P$ is the defining polynomial of $\theta$ and

$$
\operatorname{deg} \theta=\operatorname{deg} P=n, \quad \text { height } \theta=\text { height } P=\max _{0 \leqslant i \leqslant n}\left|a_{i}\right| .
$$

An algebraic number is an algebraic integer if its defining polynomial is monic, that is $a_{n}=1$. The denominatior of $\theta$ is the smallest positive integer, $a$, so that $a \theta$ is an algebraic integer. The denominator is a divisor of $a_{n}$.

The conjugates of $\theta$ are the zeros $\theta^{(1)}=\theta, \theta^{(2)}, \ldots, \theta^{(n)}$ of $P$. The norm of $\theta$ is

$$
\operatorname{norm} \theta=\theta^{(1)} \theta^{(2)} \cdots \theta^{(n)}=(-1)^{n} a_{0} / a_{n} .
$$

An algebraic number field is a field $K=\mathbf{Q}(\theta)$ obtained by adjoining an algebraic number $\theta$ to $\mathbf{Q}$. The degree of $K$ is

$$
[K: Q]=\operatorname{deg} \theta .
$$

The elements $\alpha \in K$ can be expressed uniquely as polynomials $f(\theta)$ where $f(x) \in$ $\mathbf{Q}[x], \operatorname{deg} f \leqslant[K: Q]$. The $K$-conjugates of $\alpha$ are $f\left(\theta^{(1)}\right), f\left(\theta^{(2)}\right), \ldots, f\left(\theta^{(n)}\right)$ which are not necessarily distinct and the $K$-norm of $\alpha$ is

$$
\operatorname{norm}_{K / Q} \alpha=f\left(\theta^{(1)}\right) f\left(\theta^{(2)}\right) \cdots f\left(\theta^{(n)}\right) .
$$

Treating the conjugates of $\theta$ as complex numbers we define the house of $\theta$,

$$
|\theta|=\max _{1 \leqslant i \leqslant n}\left|\theta^{(i)}\right| \text {. }
$$

Finally a few simple observations.

(i) Irreducibility in $\mathbf{Z}[x, y]$ means not only irreducibility of the polynomial in $\mathbf{Q}[x, y]$ but also that the coefficients are relatively prime.

(ii) For two polynomials $f(x), g(x)$ we have

$$
\operatorname{height}(f g) \leqslant(1+\operatorname{deg} f) \operatorname{height}(f) \cdot \operatorname{height}(g) \text {. }
$$

(iii) If $P(x) \in \mathbf{C}[x]$ and $P(\theta)=0$ then

$$
|\theta|<\text { height } P+1 \text {. }
$$

(iv) A polynomial $P\left(x_{1}, x_{2}, \ldots, x_{n}\right) \in \mathbf{Z}\left[x_{1}, x_{2}, \ldots, x_{n}\right]$ which is symmetric in $x_{1}, x_{2}, \ldots, x_{n}$ is equal to a polynomial $Q\left(\sigma_{1}, \sigma_{2}, \ldots, \sigma_{n}\right) \in \mathbf{Z}\left[\sigma_{1}, \sigma_{2}, \ldots, \sigma_{n}\right]$ in the elementary symmetric functions

$$
\sigma_{1}=x_{1}+x_{2}+\cdots+x_{n}, \sigma_{2}=x_{1} x_{2}+x_{1} x_{3}+\cdots+x_{n-1} x_{n}, \ldots, \sigma_{n}=x_{1} x_{2} \cdots x_{n} .
$$

(v) Hadamard's inequality. If $A=\left(a_{i j}\right)_{i, j=1,2, \ldots, n}$ is an $n \times n$ complex matrix then

$$
|\operatorname{det} A| \leqslant\left(\prod_{i=1}^{n} \sum_{j=1}^{n}\left|a_{i j}\right|^{2}\right)^{1 / 2} \text {. }
$$


(vi) The notation $f(x)=O(g(x))$ means that $|f(x)| \leqslant c|g(x)|$ for some constant $c$ and all large $|x|$. The symbol

$$
\sum a_{i} x^{i} \ll \sum b_{i} x^{i}
$$

means $\left|a_{i}\right| \leqslant b_{i}$ for all $i$.

For more on such algebraic concepts, see LeVeque [7, Volume II] and Pollard and Diamond [11].

\section{Qualitative results.}

2.1. THEOREM. Let $y=f(x)$ where $f$ is a function of a complex variable $x$ given by a Puiseux series

$$
f(x)=\sum_{n=-m}^{\infty} a_{n} x^{-n / e}, \quad m, e \in \mathbf{Z}, a_{-m} \neq 0, e>0
$$

which converges for $|x|>R$. let $a_{n} \in K$, where $K$ is an algebraic number field of degree $s$, for all $n \leqslant N$ and let $M$ be the number of pairs of integers $(\mu, \nu)$ with

$$
1 \leqslant \mu \leqslant \nu m / e, \quad 1 \leqslant \nu<s e .
$$

If

$$
N \geqslant M e+(s e-1) m,
$$

then the lattice points $(x, y)$ on the curve $y=f(x)$ satisfy an equation $P(x, y)=0$ where $P(x, y) \in \mathbf{Z}[x, y], \operatorname{deg}_{y} P \leqslant s e$, and $P_{\lambda}$ is a monomial for all $\lambda \neq m / e$. For $\lambda=m / e, P_{\lambda}$ is a constant multiple of a power of $x$ times a power of an irreducible polynomial in $\mathbf{Z}[x, y]$. Hence, $\tilde{P}=P_{m / e}$. If the number of lattice points on $y=f(x)$ is infinite, then $P(x, f(x)) \equiv 0$.

Proof. To exclude trivialities we observe that if $m<0$ then $f(x) \rightarrow 0$ as $x \rightarrow \infty$. Thus for large $x$ the only lattice points on $y=f(x)$ are given by the integral zeros of $f(x)$, and since $f(x)$ is not identically zero these are finite in number. If $m=0$ then $f(x) \rightarrow a_{0}$ as $x \rightarrow \infty$ and either $f(x) \equiv a_{0} \in \mathbf{Z}$ or, again, there are are only finitely many lattice points on $y=f(x)$. We, therefore, restrict our attention to the case $m>0$ and hence $N \geqslant 0$.

Let $a_{n}^{(\sigma)} ; \sigma=1,2, \ldots, s$; denote the conjugates of $a_{n}=a_{n}^{(1)}, n \leqslant N$ and let $\zeta=$ $\exp (2 \pi i / e)$. Consider the functions

$$
F(x, y ; \rho)=x^{\rho} \prod_{\sigma=1}^{s} \prod_{\varepsilon=0}^{e-1}\left(y-\sum_{n=-m}^{N} a_{n}^{(\sigma)}\left(\zeta^{\varepsilon} x^{-1 / e}\right)^{n}\right), \quad \rho=0,1, \ldots, M
$$

For $y=f(x)$ we have

$$
y-\sum_{n=-m}^{N} a_{n}^{(\sigma)}\left(\zeta^{\varepsilon} x^{-1 / e}\right)^{n}= \begin{cases}O\left(x^{-(N+1) / e}\right) & \text { for } \sigma=1, \varepsilon=0, \\ O\left(x^{m / e}\right) & \text { otherwise. }\end{cases}
$$

Thus $F(x, y ; \rho)=O\left(x^{k}\right)$ where

$$
k \leqslant M+(s e-1) m / e-(N+1) / e \leqslant-1 / e
$$

so that

$$
F(x, y ; \rho)=O\left(x^{-1 / e}\right), \quad \rho=0,1, \ldots, M
$$


Now, from (2.4) we see that

$$
F(x, y ; \rho)=P(x, y ; \rho)+\sum_{\mu} \sum_{\nu} b_{\rho \mu \nu} y^{\nu} / x^{\mu}+O\left(x^{-1 / e}\right)
$$

where $P(x, y ; \rho) \in \mathbf{Q}[x, y]$ and the double sum is extended over all $\mu, \nu$ which satisfy (2.3). The $b_{\rho \mu \nu}$ are rational numbers. The terms collected in $O\left(x^{-1 / e}\right)$ are terms of the form $y^{\nu} / x^{\mu}$ with $m \nu / e<\mu \leqslant N s, 0 \leqslant \nu \leqslant s e$.

Since there are $M+1$ functions $F(x, y ; \rho)$, there exist integers $B_{\rho}$, not all zero, so that

$$
\sum_{\rho=0}^{M} B_{\rho} b_{\rho \mu \nu}=0
$$

for all pairs $\mu, \nu$ which satisfy (2.3), and so that

$$
Q(x, y)=\sum_{\rho=0}^{M} B_{\rho} P(x, y ; \rho)=\left(\sum_{\rho=0}^{M} B_{\rho} x^{\rho}\right) y^{s e}+\cdots \in \mathbf{Z}[x, y] .
$$

From (2.7), (2.8), and (2.9) we get

$$
\sum_{\rho=0}^{M} B_{\rho} F(x, y ; \rho)=Q(x, y)+O\left(x^{-1 / e}\right) .
$$

The lattice points $(x, y)$ on $y=f(x)$ satisfy (2.6), and so by (2.10) they satisfy

$$
Q(x, y)=O\left(x^{-1 / e}\right) \text {. }
$$

Since $Q(x, y)$ is an integer, it follows from (2.11) that $Q(x, y)=0$ for all lattice points with large $|x|$. For the finitely many $x$-coordinates of the remaining lattice points there is a polynomial $Q_{1}(x) \in \mathbf{Z}[x]$ so that $Q_{1}(x)=0$ for all these values. Hence $P(x, y)=Q_{1}(x) Q(x, y)$ vanishes at all lattice points of (2.2).

If there are infinitely many lattice points then the analytic function $P(x, f(x))$ has infinitely many zeros in any neighborhood of $\infty$, while the point at $\infty$ is an algebraic singularity. Hence $P(x, f(x)) \equiv 0$.

Write $m / e=m_{1} / e_{1}$ where $\left(m_{1}, e_{1}\right)=1$, and let $g(x)=0$ be the defining equation of $a_{-m}^{e_{1}}$ with degree $s_{1}$ and leading coefficient $b$. Then

$$
G(x, y)=x^{m_{1} s_{1}} g\left(y^{e_{1}} / x^{m_{1}}\right)
$$

is irreducible in $\mathbf{Z}[x, y]$ and

$$
P_{m / e}(x, y ; \rho)=\tilde{P}(x, y ; \rho)=c x^{\rho} G(x, y)^{e s / e_{1} s_{1}},
$$

where $c=b^{-e s / e_{1} s_{1}}$. It follows that if $\rho_{1}$ is the largest index for which $B_{\rho_{1}} \neq 0$, then

$$
Q_{m / e}(x, y)=\tilde{Q}(x, y)=c B_{\rho_{1}} x^{\rho_{1}} G(x, y)^{e s / e_{1} s_{1}}
$$

and

$$
\tilde{P}(x, t)=c B_{1} x^{\rho_{1}} \tilde{Q}_{1}(x) G(x, y)^{e s / e_{1} s_{1}} .
$$

This completes the proof of the theorem.

2.12. Theorem (Runge). Let $F(x, y)$ satisfy Runge's Condition. Then the Diophantine equation $F(x, y)=0$ has only finitely many solutions $(x, y) \in \mathbf{Z}^{2}$. 
Proof. If $\operatorname{deg} F=1$, then the theorem is true because Runge's Condition is violated. We, therefore, assume that $\operatorname{deg} F>1$. If $F(x, y)=0$ has infinitely many integral solutions then there are infinitely many lattice points on one of the expansions $y=f(x)$ of $y$ into a Puiseux series at $\infty$. By Theorem 2.1 this implies that $P(x, f(x))=0$ where $P$ is the polynomial determined in Theorem 2.1. Since $F$ is irreducible it must divide $P$ and thus $F_{\lambda}$ divides $P_{\lambda}$ for all $\lambda$.

Hence $F_{\lambda}$ is a monomial except possibly for a single value $\lambda=\lambda_{0}$, and $F_{\lambda_{0}}$ is a constant times a power of $x$ times a power of the irreducible $G(x, y)$ of Theorem 2.1 .

Reversing the role of $x$ and $y$ we see that $F_{\lambda_{0}}$ is a constant multiple of a power of $y$ times a power of $G(x, y)$. Thus $F_{\lambda_{0}}$ is either a monomial or a constant multiple of a power of $G(x, y)$.

If $F_{\lambda}$ is a monomial for all $\lambda$ then $\tilde{F}$ is a monomial. If $|x|,|y|$ are both large then a monomial $\tilde{F}$ dominates the rest of $F$. Thus $F=0$ can have infinitely many solutions in this case only when $F=x-a$ or $F=y-b$, that is, when $\operatorname{deg} F=1$. Therefore $\tilde{F}=F_{\lambda_{0}}=a G(x, y)^{b}$ where $a, b$ are positive integers.

Note that Runge's Condition is not an invariant under changes of variables which preserve lattice points. Thus, say,

$$
F(x, y)=\left(x+y^{2}\right)^{2}-y^{2}+a y+b
$$

has leading part $\left(x+y^{2}\right)^{2}$ which is a power of an irreducible polynomial. But, if we set $x^{\prime}=x+y^{2}, y^{\prime}=y$ then $F(x, y)=G\left(x^{\prime}, y^{\prime}\right)=x^{\prime 2}-y^{\prime 2}+a y^{\prime}+b$ where $\tilde{G}=$ $\left(x^{\prime}+y^{\prime}\right)\left(x^{\prime}-y^{\prime}\right)$, so that $G$ satisfies Runge's Condition. It would be interesting to characterize the class of polynomials which are equivalent to polynomials satisfying Runge's Condition under lattice-point preserving changes of variable.

All results in this section remain valid if we replace lattice points by lattice points over imaginary quadratic number fields. For lattice points over number fields with more than one Archimedean valuation we need additional hypotheses.

We use the following notation: Let $L$ denote an algebraic number field of degree $t$, and let $\theta_{L}$ denote the ring of integers of $L$. For $x \in L$, we have the conjugates $x^{(\tau)}$, $\tau=1,2, \ldots, t$. A pair $(x, y) \in \mathcal{O}_{L}^{2}$ is an L-lattice point.

2.13. TheOREM. Let $f(x)$ be as in Theorem 2.1. Consider the L-lattice points $(x, y)$ with the following properties:

(i) $y^{\left(\tau_{0}\right)}=f\left(x^{\left(\tau_{0}\right)}\right)$ for some $\tau_{0}$ with $\left|x^{\left(\tau_{0}\right)}\right| \geqslant|x|^{c_{1}}$,

(ii) $\left\lceil\mid \leqslant c_{2}\left\lceil\left. x\right|^{c_{3}}\right.\right.$,

(iii) $|1 / x| \leqslant c_{4}|\bar{x}|^{s}$,

where $c_{1}, c_{2}, c_{3}, c_{4}$ and $c_{5}$ are positive constants with

$$
c_{5}<c_{1} / e s(t-1) \text {. }
$$

Let $M$ be as in Theorem 2.1. Let

$$
N \geqslant(e+(s e-1) m) /\left(c_{1}-c_{5} s e(t-1)\right), \quad N \geqslant c_{3} e / c_{5}, \quad N \geqslant m / c_{5} .
$$

Then all the above L-lattice points satisfy an equation $P(x, y)=0$, where $P$ is a polynomial with the properties given in Theorem 2.1. If the number of L-lattice points satisfying (i)-(iii) is infinite, then $P(x, f(x)) \equiv 0$. 
Note that Theorem 2.13 contains Theorem 2.1 as the special case $t=1, c_{1}=1$, $c_{3}=m / e, c_{5}=m / N$.

Proof. Construct the functions $F(x, y ; \rho)$ of $(2.4)$. For $\left|x^{\left(\tau_{0}\right)}\right|>R$. Condition (2.5) becomes

$$
y^{\left(\tau_{0}\right)}-\sum_{n=-m}^{N} a_{n}^{(\sigma)}\left(\zeta^{\varepsilon}\left(x^{\left(\tau_{0}\right)}\right)^{-1 / e}\right)^{n}= \begin{cases}O\left(|\sqrt{x}|^{-c_{1}(N+1) / e}\right) & \text { for } \sigma=1, \varepsilon=0, \\ O\left(|x|^{m / e}\right) & \text { otherwise. }\end{cases}
$$

Thus

$$
F\left(x^{\left(\tau_{0}\right)}, y^{\left(\tau_{0}\right)} ; \rho\right)=O\left(|\bar{x}|^{k}\right), \quad \rho=0,1, \ldots, M
$$

where

$$
k \leqslant M+(s e-1) m / e-c_{1}(N+1) / e .
$$

For arbitrary $\tau$ we have from (ii) and (iii),

$$
\begin{aligned}
y^{(\tau)}-\sum_{n=-m}^{N} a_{n}^{(\sigma)}\left(\zeta^{\varepsilon}\left(x^{(\tau)}\right)^{-1 / e}\right)^{n} & =O\left(|x|^{c_{3}}+|x|^{m / e}+\left\lceil\left. x\right|^{c_{5} N / e}\right)\right. \\
& =O\left(|\bar{x}|^{c_{5} N / e}\right),
\end{aligned}
$$

and hence

$$
F\left(x^{(\tau)}, y^{(\tau)} ; \rho\right)=O\left(|x|^{c_{s} s N}\right) .
$$

Now construct $Q(x, y)$ as in (2.9) to get

$$
Q\left(x^{\left(\tau_{0}\right)}, y^{\left(\tau_{0}\right)}\right)=O\left(|x|^{k}\right), \quad Q\left(x^{(\tau)}, y^{(\tau)}\right)=O\left(|x|^{c_{S} s N}\right) .
$$

Thus

$$
\operatorname{norm} Q(x, y)=O\left(\widehat{x}^{k+c_{5} s N(t-1)}\right)=O\left(\left.{ }_{x}\right|^{-c_{1} / e}\right),
$$

and consequently $Q(x, y)=0$ for all but a finite number of our $L$-lattice points. The construction of $P(x, y)$ now proceeds as in the proof of Theorem 2.1. If there are infinitely many such $L$-lattice points then $|x|$, and hence $x^{\left(\tau_{0}\right)}$, tend to infinity so that $P(x, f(x))$ has infinitely many zeros in every neighborhood of $\infty$. Thus $P(x, f(x)) \equiv 0$.

We can now apply Theorem 2.13 to the following generalization of Runge's Theorem.

2.14. THEOREM. Let $F(x, y)$ be irreducible in $\mathbf{Z}[x, y]$ and set

$$
F(x, y)=\sum_{i=0}^{d_{1}} x^{i} f_{i}(y)=\sum_{j=0}^{d_{2}} y^{j} g_{j}(x)
$$

where

$$
g_{d_{2}}(x)=a \prod_{r=1}^{v}\left(x-\alpha_{r}\right)^{\mu_{r}}, \quad f_{d_{1}}(y)=b \prod_{s=1}^{w}\left(y-\beta_{s}\right)^{\nu}
$$

and assume that there exist infinitely many L-lattice points $(x, y) \in \Theta_{L}^{2}$ which satisfy $F(x, y)=0$ and

$$
|1 / x| \leqslant c_{6}|x|^{1 /(t-1) d_{2}^{2}}, \quad\left\lceil 1 /\left.y\left|\leqslant c_{6}\right| y\right|^{1 /(t-1) d_{1}^{2}}\right.
$$


where $c_{6}$ is a positive constant. Assume one of the following conditions for these L-lattice points:

$$
\begin{array}{ll}
\left|1 /\left(x-\alpha_{r}\right)\right| \leqslant c_{7}|y|^{1 / \mu_{r}-c_{8}}, & r=1,2, \ldots, v, \\
1 /\left.\left(y-\beta_{s}\right)\left|\leqslant c_{7}\right| x\right|^{1 / \nu_{s}-c_{8}}, & s=1,2, \ldots, w,
\end{array}
$$

where $c_{7}, c_{8}$ are positive constants. Then $F(x, y)$ violates Runge's Condition.

Proof. Assume that there are infinitely many $L$-lattice points $(x, y)$ which satisfy the hypotheses of the theorem. Then, since there are only finitely many integers $\theta$ in ${ }^{(}{ }_{L}$, with $|\theta|$ below a fixed bound, we have $\left.\mid x\right\rceil \rightarrow \infty$ and $|y| \rightarrow \infty$. Since $F\left(x^{(\tau)}, y^{(\tau)}\right)$ $=0$ for $\tau=1,2, \ldots, t$ we can pick $\tau_{0}$ so that $\left|x^{\left(\tau_{0}\right)}\right|=|x|$ for infinitely many values of $x$. We can also pick one Puiseux series expansion $y=f(x)$ at $\infty$ with $y^{\left(\tau_{0}\right)}=f\left(x^{\left(\tau_{0}\right)}\right)$ for infinitely many of those values of $x$. If we restrict attention to that series and to those $L$-lattice points then (i) of Theorem 2.13 is satisfied with $c_{1}=1$. (iii) of Theorem 2.13 follows from (iv) since the degree $s$ of the field of Puiseux series coefficients of $f$ satisfies $s \leqslant d_{2}$, and since $e \leqslant d_{2}$, where this last inequality is strict if Runge's Condition holds. To verify (ii) we note that (v) implies

$$
|y|^{d_{2}+c_{9}-1}=O\left(|y|^{d_{2}-1} \cdot|x|^{d_{1}}\right)
$$

and hence

$$
\bar{y} \mid=O\left(|x|^{d_{1} / c_{8}}\right)
$$

which implies (ii). Thus, according to Theorem 2.13 we have an equation $P(x, f(x))$ $\equiv 0$ where $\tilde{P}(x, y)$ is a constant times a power of $x$ times a power of an irreducible polynomial.

We can now interchange the role of $x$ and $y$ to get that $\tilde{F}$ divides both a constant times a power of $x$ times a power of an irreducible polynomial and a constant times a power of $y$ times a power of an irreducible polynomial. Thus, in order to show that $F$ violates Runge's Condition it suffices to show that $\tilde{F}$ is not a monomial.

Now, if $\tilde{F}=a_{d_{1} d_{2}} x^{d_{1}} y^{d_{2}}$ then

$$
\left|x^{(\tau)}\right|^{d_{1}}\left|y^{(\tau)}\right|^{d_{2}}=O\left(\left|x^{(\tau)}\right|^{d_{1}}\left|y^{(\tau)}\right|^{d_{2}-1}+\left|x^{(\tau)}\right|^{d_{1}-1}\left|y^{(\tau)}\right|^{d_{2}}\right) .
$$

Thus not both $\left|x^{(\tau)}\right|$ and $\left|y^{(\tau)}\right|$ can be large. Since we are interested in solutions with $\left|x^{\left(\tau_{0}\right)}\right|=|x| \rightarrow \infty$ we must have $y^{\left(\tau_{0}\right)}$ bounded. Now (v) implies

$$
\left.{ }_{x}\right|^{d_{1}+c_{8}-1}=O\left(|\bar{x}|^{d_{1}-1}\right)
$$

which is incompatible with the unboundedness of $\mid x\rceil$.

(v) in Theorem 2.14 is necessary. For example, the equation $(x-a)(y-b)=1$ violates Runge's Condition, but has infinitely many $L$-lattice point solutions $x=a$ $+\eta, y=b+\eta^{-1}$, in any field $L$ with an infinite group of units $\eta$.

3. Quantitative results. As before we consider Diophantine equations

$$
F(x, y)=0, \quad(x, y) \in \mathbf{Z}^{2},
$$

where $F$ is an irreducible polynomial in $\mathbf{Z}[x, y]$ and

$$
F=\sum_{i=0}^{d_{1}} \sum_{j=0}^{d_{2}} a_{i j} x^{i} y^{j},
$$


$d_{1}=\operatorname{deg}_{x} F, d_{2}=\operatorname{deg}_{y} F, d=\max \left\{d_{1}, d_{2}\right\}, h=$ height $F$.

Equation (3.1) defines $y$ as an algebraic function of the complex variable $x$. The irreducibility of $F$ implies that the resultant $\operatorname{res}_{y}\left(F, F_{y}\right)$ of $F$ and $F_{y}=\partial F / \partial y$ is a polynomial in $x$ which does not vanish identically. The finite singularities $x$ of $y$

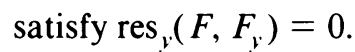

In this section we compute bounds for certain equations (3.1) that satisfy Runge's Condition. We begin with some simple cases.

3.2. THEOREM. If $\tilde{F}=a_{d_{1} d_{2}} x^{d_{1}} y^{d_{2}}$ is a monomial, $d_{1}, d_{2}>0$, then the integral solutions of (3.1) satisfy

$$
\max \{|x|,|y|\} \leqslant(2(h+1))^{d+1} .
$$

Proof. For $|x|>1,|y|>1$, we have

$$
\begin{aligned}
&|F(x, y)| \geqslant\left|a_{d_{1} d_{2}} x^{d_{1}} y^{d_{2}}\right|-\sum_{(i, j) \neq\left(d_{1}, d_{2}\right)}\left|a_{i j} x^{i} y^{j}\right| \\
& \geqslant|x|^{d_{1}}|y|^{d_{2}}-h\left(\sum_{i=0}^{d_{1}}\left|x^{i}\right| \cdot \sum_{j=0}^{d_{2}}\left|y^{j}\right|-|x|^{d_{1}}|y|^{d_{2}}\right) \\
&=(h+1)|x|^{d_{1}}|y|^{d_{2}}-h \frac{|x|^{d_{1}+1}-1}{|x|-1} \frac{|y|^{d_{2}+1}-1}{|y|-1} \\
&>\frac{|x|^{d_{1}}|y|^{d_{2}}}{(|x|-1)(|y|-1)}(|x||y|-(h+1)(|x|+|y|)) .
\end{aligned}
$$

Thus $|F(x, y)|>0$ if $|x|,|y| \geqslant 2(h+1)$. If we assume that $\left|x_{0}\right|<2(h+1)$ then the equation $F\left(x_{0}, y\right)=0$ is an equation for $y$ which does not hold identically and has height less than $(2(h+1))^{d+1}$. Hence all solutions satisfy $|y| \leqslant(2(h+1))^{d+1}$. Similarly, if $\left|y_{0}\right|<2(h+1)$ then the solutions of $F\left(x, y_{0}\right)=0$ satisfy $|x| \leqslant$ $(2(h+1))^{d+1}$.

3.3. TheOREM. If $\tilde{F}$ is reducible in $\mathbf{Z}[x, y]$, and if either $d_{1}=1$ or $d_{2}=1$, then the integral solutions of (3.1) satisfy

$$
\max \{|x|,|y|\} \leqslant d(h+1)^{2 d} .
$$

Note that Runge's Condition is equivalent to the reducibility of $\tilde{F}$ in this case.

Proof. Assume $d_{2}=1$. Then $y$ is a rational function of $x$ which is not a polynomial. We set $y=P(x) / Q(x)$ where $P(x), Q(x) \in \mathbf{Z}[x]$ and where

$$
Q(x)=b_{0}+b_{1} x+\cdots+b_{q} x^{q}, \quad\left|b_{i}\right| \leqslant h,(0 \leqslant i \leqslant q), b_{q}>0, q \leqslant d .
$$

If we write $X=b_{q} x$ then

$$
Q(x)=b_{q}^{-q+1}\left(B_{0}+B_{1} X+\cdots+B_{q-1} X^{q-1}+X^{q}\right)
$$

where $B_{i}=b_{i} b_{q}^{q-1-i} ; i=0,1, \ldots, q$. Hence

$$
\begin{aligned}
Q(x)^{-1} & \ll b_{q}^{q-1} X^{-q}\left[1-h\left(b_{q} X^{-1}+b_{q}^{2} X^{-2}+\cdots+b_{q}^{q} X^{-q}\right)\right]^{-1} \\
& \ll b_{q}^{q-1} X^{-q} /\left(1-(h+1) b_{q} X^{-1}\right)=b_{q}^{-1} x^{-q} /(1-(h+1) / x) .
\end{aligned}
$$


Since the coefficients in the Laurent series of $Q(x)^{-1}$ in terms of $X$ are integers divisible by $b_{q}^{q-1}$, it follows that the coefficient of $x^{-j}, j \geqslant q$, in the Laurent series of $Q(x)^{-1}$ in terms of $x$ is a rational number with denominator $b_{q}^{j-q+1}$.

We now write

$$
P(x)=a_{0}+a_{1} x+\cdots+a_{p} x^{p} \ll h\left(1+x+\cdots+x^{d}\right) .
$$

Thus the nonnegative powers of $x$ in the Laurent series of $P / Q$ form a polynomial $b_{q}^{-d+q-1} R(x)$ where $R(x) \in \mathbf{Z}[x], \operatorname{deg} R<d$ and

$$
\text { height } R \leqslant b_{q}^{d-q}(h+1)^{d} \text {. }
$$

The negative powers in the Laurent series $P / Q$ form a fraction $S / Q$ where

$$
S=P-b_{q}^{-d+q-1} Q R \text {. }
$$

Thus $b_{q}^{d-q+1} S \in \mathbf{Z}[x]$ and

$$
\text { height }\left(b_{q}^{d-q+1} S\right) \leqslant b_{q}^{d-q}\left(b_{q} h+d h(h+1)^{d}\right) .
$$

Now, if $x, y$ are integers then $b_{q}^{d-q+1} S(x) / Q(x)$ is an integer. Thus either $S(x)=0$, which according to (3.4) implies

$$
|x| \leqslant b_{q}^{d-q} d(h+1)^{d+1}<d(h+1)^{2 d},
$$

or $|Q(x)| \leqslant b_{q}^{d-q+1}|S(x)|$, which yields

$$
b_{q}|x|^{q}-h \frac{|x|^{q}-1}{|x|-1} \leqslant|Q(x)| \leqslant b_{q}^{d-q+1}|S(x)| \leqslant b_{q}^{d-q} d(h+1)^{d+1} \frac{|x|^{q}-1}{|x|-1} .
$$

Hence

$$
|x| \leqslant b_{q}^{-1}\left(b_{q}^{d-q} d(h+1)^{d+1}+h\right)+1<d(h+1)^{2 d} .
$$

Therefore attention can now be restricted to the case that $d_{1}, d_{2}>1$ and $\tilde{F}$ is not a monomial. This general case requires an analysis of the Puiseux series expansions of $y$. Such Puiseux series converge and represent $y$ for all finite values of $x$ in the exterior of any circle about the origin that encloses all finite singularities of $y$.

3.5. Lemma. An algebraic function $y$ defined by (3.1) has no singularities with $R_{0} \leqslant|x|<\infty$, where

$$
R_{0}=4^{d} d^{4 d} h^{2 d-1} .
$$

Proof. Let $x$ be a finite singularity of $y$. Then

$$
D(x)=\operatorname{res}_{y}\left(F, F_{y}\right)=0 .
$$

Here $D(x)$ is an $n \times n$ determinant, with $n \leqslant 2 d-1$, whose nonzero entries are the coefficients of the powers of $y$ in $F$ and $F_{y}$. Thus the entries are polynomials of degree at most $d$, and height at most $h$ for the coefficients of $F$ and at most $d h$ for the coefficients of $F_{y}$. So, using Hadamard's inequality, we have

$$
D(x) \ll(2 d-1)^{d-1 / 2} d^{d} h^{2 d-1}\left(1+x+\cdots+x^{d}\right)^{2 d-1} .
$$

Therefore

$$
\text { height } D \leqslant(2 d-1)^{d-1 / 2} d^{d} h^{2 d-1}(1+d)^{2 d-1}<4^{d} d^{4 d} h^{2 d-1} .
$$


Now $D(x)=0$ implies

$$
|x|<\text { height } D+1 \leqslant 4^{d} d^{4 d} h^{2 d-1} \text {. }
$$

Let

$$
y=\sum_{n=-m}^{\infty} a_{n} x^{-n / e}
$$

be a Puiseux series for a function defined by (3.1), with coefficients $a_{n}$ in a field $K$, $[K: \mathbf{Q}]=s$. Since all the conjugate series

$$
y(x, \sigma, \varepsilon)=\sum_{n=-m}^{\infty} a_{n}^{(\sigma)}\left(\zeta^{\varepsilon} x^{-1 / e}\right)^{n}
$$

$\sigma=1,2, \ldots, s ; \varepsilon=0,1, \ldots, e-1 ; \zeta=\exp (2 \pi i / e)$, are also solutions of $(3.1)$, it follows that the number of distinct series (3.8) is at most $d_{2}$. Moreover, it follows from Runge's Condition, by employing an argument similar to the proof of Theorem 2.12 , that the number of distinct series (3.8) is, in fact, less than $d_{2} \leqslant d$. In particular it follows, as pointed out earlier, that $e<d_{2}$. We assume that Runge's Condition holds for the Diophantine equation (3.1) in the computation of the bounds to follow.

We now normalize (3.7) by setting $x=t^{-e}, y=z / t^{m}$. Then the series

$$
z=\sum_{n=0}^{\infty} b_{n} t^{n}, \quad b_{n}=a_{n-m},
$$

satisfies the equation

$$
H(t, z)=t^{k} F\left(t^{-e}, z / t^{m}\right)=0,
$$

where $k \leqslant d(e+m)$ is chosen so that $H$ is a polynomial and $H(0, z) \neq 0$. Since $1 \leqslant e \leqslant d$ and $0 \leqslant m \leqslant d$ (the case $m<0$ is trivial), it follows that

$$
\text { height } H=h, \quad \operatorname{deg}_{t} H<2 d^{2}, \quad \operatorname{deg}_{z} H \leqslant d .
$$

Moreover, $z$ is analytic for $|t| \leqslant R_{0}^{-1 / e}$ where $R_{0}$ is given by (3.6).

Each of the formal power series

$$
z_{\sigma}=\sum_{n=0}^{\infty} b_{n}^{(\sigma)} t^{n}, \quad \sigma=1,2, \ldots, s,
$$

is analytic for $|t| \leqslant R_{0}^{-1 / e}$. Thus

$$
\left|b_{n}^{(\sigma)}\right|=\left|\frac{1}{2 \pi i} \oint_{|r|=r} \frac{z_{\sigma}(t)}{t^{n+1}} d t\right| \leqslant \frac{1}{r^{n}} \max _{|r|=r}\left|z_{\sigma}(t)\right|, \quad r \leqslant R_{0}^{-1 / e} .
$$

In order to estimate $z_{\sigma}(t)$ we write

$$
H(t, z)=A_{0}(t)+A_{1}(t) z+\cdots+A_{d_{2}}(t) z^{d_{2}} .
$$

Then $\operatorname{deg} A_{i}(t)<2 d^{2}$, height $A_{i} \leqslant h$ and, since $r \leqslant(4 h)^{-1}$, we have

$$
\max _{|t|=r}\left|A_{i}(t)\right|<h+1, \quad i=0,1, \ldots, d_{2} .
$$

Also, writing $A_{d_{2}}(t)=t^{l} B(t)$ with $B(0) \neq 0$ we have

$$
\min _{|t| c \mid}\left|A_{d_{2}}(t)\right| \geqslant r^{l} \min _{|t|=r} B(t)>\frac{1}{2} r^{2 d^{2}} \text {. }
$$


Thus, if we divide (3.11) by $A_{d_{2}}(t)$ we get a monic equation for $z_{\sigma}(t)$ of height at most $2(h+1) r^{-2 d^{2}}-1$. Hence

$$
\max _{|t|=r}\left|z_{\sigma}(t)\right|<2(h+1) r^{-2 d^{2}}
$$

and (3.10) yields

$$
\left|b_{n}^{(\sigma)}\right| \leqslant 2(h+1) r^{-2 d^{2}-n} .
$$

If we choose $r=R_{0}^{-1 / e}$ we get

$$
\mid b_{n} \leqslant 2(h+1) R_{0}^{2 d^{2} / e} R_{0}^{n / e}=C_{1} R_{1}^{n}
$$

where

$$
C_{1}=2(h+1) R_{0}^{2 d^{2} / e}, \quad R_{1}=R_{0}^{1 / e} .
$$

Substitution of (3.12) in the Puiseux series (3.8) yields

$$
y(x ; \sigma, \varepsilon) \ll C_{1} R_{1}^{m} \sum_{n=-m}^{\infty}\left(R_{1} x^{-1 / e}\right)^{n}=C_{1} x^{m / e} /\left(1-\left(R_{0} / x\right)^{1 / e}\right) .
$$

In order to get a better quantitative estimate we now modify the definition (2.4) of $F(x, y ; \rho)$. Let $\rho=0,1, \ldots, 2 M$ and construct the functions $F(x, y ; \rho)$ from an algebraic function $y$ given by (3.1). We let $N=\infty$ since now all of the Puiseux coefficients $a_{n}$ are in an algebraic number field. Let

$$
F(x, y ; \rho)=x^{\rho} \Pi\left(y-\sum_{n=-m}^{\infty} a_{n}^{(\sigma)}\left(\zeta^{\varepsilon} x^{-1 / e}\right)^{n}\right),
$$

where the product is extended over the less than $d$ series (3.8) that are distinct.

By using (3.13) we get

$$
\begin{aligned}
F(x, y ; \rho) & \ll x^{\rho}\left(y+C_{1} x^{m / e}\left(1-\left(R_{0} / x\right)^{1 / e}\right)^{-1}\right)^{d-1} \\
& \ll \sum_{\delta=0}^{d-1} y^{\delta}\left(\begin{array}{l}
d \\
\delta
\end{array}\right) C_{1}^{d-\delta} x^{\rho+(d-\delta) m / e}\left(1-\left(R_{0} / x\right)^{1 / e}\right)^{\delta-d} \\
& \ll\left(2 C_{1}\right)^{d} \sum_{\delta=0}^{d} y^{\delta} x^{\rho+(d-\delta) m / e}\left(1-\left(R_{0} / x\right)^{1 / e}\right)^{-d} \\
& \ll\left(2 C_{1}\right)^{d} \sum_{\delta=0}^{d} y^{\delta} x^{\rho+(d-\delta) m / e} \sum_{n=0}^{\infty}\left(\begin{array}{c}
d+n-1 \\
d-1
\end{array}\right)\left(R_{0} / x\right)^{n / e} \\
& \ll\left(4 C_{1}\right)^{d} \frac{x^{\rho+d m / e}}{1-2\left(R_{0} / x\right)^{1 / e}} \sum_{\delta=0}^{d} y^{\delta} x^{-\delta m / e} .
\end{aligned}
$$

3.16. LEMMA. The coefficient $b_{0}=a_{-m}$ in (3.9) is algebraic and satisfies deg $b_{0} \leqslant d$, $b_{0}<h / D_{0}+1, D_{0} b_{0}$ is an algebraic integer where $D_{0}$ is a positive integer, with $D_{0} \leqslant h$.

Proof. We have the nontrivial equation $H\left(0, b_{0}\right)=0$ whose degree is at most $d$ and whose leading coefficient is $D_{0} \leqslant h$. The roots $b_{0}^{(i)}$ then satisfy $\left|b_{0}^{(i)}\right|<h / D_{0}+1$. 
In the general case the computation of the denominators of $b_{n}$ involve difficulties which we defer to $\S 4$. In this section we shall be concerned with the following case.

3.17. Assumption. Let $\beta=H_{z}\left(0, b_{0}\right) \neq 0$.

3.18. LEMMA. Let $\theta$ be an algebraic number of degree $s$ and let the leading coefficient in the defining equation of $\theta$ be $D$. If $P\left(x_{1}, x_{2}, \ldots, x_{s}\right)$ is a symmetric polynomial, with integral coefficients, in $x_{1}, x_{2}, \ldots, x_{s}$ and $\operatorname{deg}_{x_{1}} P=n$ then $D^{n} P\left(\theta^{(1)}, \theta^{(2)}, \ldots, \theta^{(s)}\right) \in \mathbf{Z}$.

Proof. We have $P\left(x_{1}, x_{2}, \ldots, x_{s}\right)=Q\left(\sigma_{1}, \sigma_{2}, \ldots, \sigma_{s}\right)$ where $Q\left(y_{1}, y_{2}, \ldots, y_{s}\right) \in$ $\mathbf{Z}\left[y_{1}, y_{2}, \ldots, y_{s}\right]$ and $\sigma_{1}, \sigma_{2}, \ldots, \sigma_{s}$ are the elementary symmetric functions of $x_{1}, x_{2}, \ldots, x_{s}$. Since $\operatorname{deg} Q=n$ and $D \sigma_{i}\left(\theta^{(1)}, \theta^{(2)}, \ldots, \theta^{(s)}\right) \in \mathbf{Z}$ it follows that $D^{n} Q\left(\sigma_{1}, \sigma_{2}, \ldots, \sigma_{s}\right) \in \mathbf{Z}$ when $x_{i}=\theta^{(i)} ; i=1,2, \ldots, s$.

3.19. Lemma. The quantity $\beta$ of Assumption 3.17 satisfies an algebraic equation over $\mathbf{Z}$ whose leading coefficient is $D_{0}^{d-1}$ and

$$
B=|\operatorname{norm} \beta| \leqslant(d+1)^{d}(2 h)^{d^{2}} / D_{0}^{d(d-1)} .
$$

Proof. Write

$$
\beta=H_{z}\left(0, b_{0}\right)=c_{0}+c_{1} b_{0}+\cdots+c_{d-1} b_{0}^{d-1}
$$

where $c_{j} \in \mathbf{Z},\left|c_{j}\right| \leqslant(j+1) h$. Thus

$$
|\beta| \leqslant h\left(1+2\left|b_{0}\right|+\cdots+d\left|b_{0}\right|^{d-1}\right) .
$$

Now for $x>1$ we have

$$
1+2 x+\cdots+d x^{d-1}=\frac{d}{d x} \frac{x^{d+1}-1}{x-1}<(d+1) \frac{x^{d}}{x-1} .
$$

Hence it follows from Lemma 3.16 and (3.20) that

$$
\begin{aligned}
|\beta| & <h(d+1)\left(\left(h / D_{0}\right)+1\right)^{d} /\left(h / D_{0}\right)=(d+1) D_{0}\left(h / D_{0}+1\right)^{d} \\
& =(d+1) D_{0}^{-d+1}\left(h+D_{0}\right)^{d}<(d+1)(2 h)^{d} / D_{0}^{d-1},
\end{aligned}
$$

and consequently that

$$
B \leqslant|\beta|^{d}<(d+1)^{d}(2 h)^{d^{2}} / D_{0}^{d(d-1)} .
$$

3.21. Lemma. If $\beta \neq 0$ then $b_{n} \in \mathbf{Q}\left(b_{0}\right)$ for all $n=1,2, \ldots$, and $\beta^{2 n-1} b_{n}$ is $a$ polynomial, with integral coefficients, in $b_{0}$, of degree at most $(2 n-1) d$.

PROOF. Substitute $z=\sum_{n=0}^{\infty} b_{n} t^{n}$ in $H(t, z)$ and expand in powers of $t$ :

$$
H(t, z)=H_{0}+H_{1} t+\cdots+H_{n} t^{n}+\cdots .
$$

Here $H_{n}$ is a polynomial, with integral coefficients, in $b_{0}, b_{1}, \ldots, b_{n}$, where for each monomial $b_{0}^{k_{0}} b_{1}^{k_{1}} \cdots b_{n}^{k_{n}}$ we have

$$
k_{0}+k_{1}+\cdots+k_{n} \leqslant d, \quad k_{1}+2 k_{2}+\cdots+n k_{n} \leqslant n .
$$

The equations $H_{n}=0$ successively define $b_{n}$ since

$$
H_{n}=\beta b_{n}+I_{n}\left(b_{0}, b_{1}, \ldots, b_{n-1}\right)
$$

where $I_{n}$ is a polynomial with integral coefficients. 
We now proceed by induction on $n$. For $n=1$ we have

$$
\beta b_{1}+H_{t}\left(0, b_{0}\right)=0 .
$$

Thus $\beta b_{1}=-H_{t}\left(0, b_{0}\right)$ is a polynomial, with integral coefficients, of degree at most $d$ in $b_{0}$.

Now assume the lemma true for indices less than $n$. The terms in $I_{n}\left(b_{0}, b_{1}, \ldots, b_{n-1}\right)$ are integral multiplies of monomials $b_{0}^{k_{0}} b_{1}^{k_{1}}, \ldots, b_{n-1}^{k_{n-1}}$ where, by (3.22),

$$
\begin{aligned}
1 k_{1}+3 k_{2}+\cdots+(2 n-3) k_{n-1}= & 2\left(k_{1}+2 k_{2}+\cdots+(n-1) k_{n-1}\right) \\
& -\left(k_{1}+k_{2}+\cdots+k_{n-1}\right) \leqslant 2 n-2,
\end{aligned}
$$

since $k_{1}+2 k_{2}+\cdots+(n-1) k_{n-1}=n$ implies $k_{1}+k_{2}+\cdots+k_{n-1} \geqslant 2$ for all $n>1$. Thus

$$
\beta^{2 n-1} b_{n}=-\beta^{2 n-2} I_{n}\left(b_{0}, b_{1}, \ldots, b_{n-1}\right)
$$

is a sum of integral multiplies of monomials

$$
\begin{aligned}
\beta^{\prime} b_{0}^{k_{0}}\left(\beta b_{1}\right)^{k_{1}}\left(\beta^{3} b_{2}\right)^{k_{2}} & \cdots\left(\beta^{2 n-3} b_{n-1}\right)^{k_{n-1}}, \\
l & =2 n-2-\left(1 k_{1}+3 k_{2}+\cdots+(2 n-3) k_{n-1}\right) \geqslant 0,
\end{aligned}
$$

which are, by the induction hypothesis, polynomials, with integral coefficients, in $b_{0}$ of degree at most

$$
\begin{aligned}
& \left(2 n-2-1 k_{1}-3 k_{2}-\cdots-(2 n-3) k_{n-1}\right) d+k_{0} \\
& \quad+\left(1 k_{1}+3 k_{2}+\cdots+(2 n-3) k_{n-1}\right) d=(2 n-2) d+k_{0} \leqslant(2 n-1) d .
\end{aligned}
$$

We now employ a modified version of the argument in the proof of Theorem 2.1. Define the functions $F(x, y ; \rho)$, for $\rho=0,1, \ldots, 2 M$, as in (3.14). It follows from Assumption 3.17 that $a_{n} \in \mathbf{Q}\left(b_{0}\right)$, for all $n$. Now $\operatorname{deg}_{y} F(x, y ; \rho)<d$ and the quantity $M$ in (2.3) becomes the number of lattice points $(\mu, \nu)$ with

$$
1 \leqslant \mu \leqslant \nu m / e, \quad 1 \leqslant \nu<d .
$$

So

$$
M \leqslant m d(d-1) / 2 e .
$$

The estimation (2.6) is replaced by

$$
F(x, y ; \rho)=0, \quad \rho=0,1, \ldots, 2 M .
$$

Now, as in (2.7), write

$$
F(x, y ; \rho)=P(x, y, \rho)+\sum_{\mu} \sum_{\nu} b_{\rho \mu \nu} y^{\nu} / x^{\mu}+O\left(x^{-1 / e}\right) .
$$

Then the coefficients $b_{\rho \mu \nu}$ are symmetric polynomials, with integral coefficients, in the conjugates of $b_{n}$ where

$$
m+1 \leqslant n \leqslant(d-1) m+\rho e+m \leqslant d m+2 e M \leqslant m d^{2} .
$$

The degree in $b_{n}$ is at most $e$. Thus, according to Lemma 3.21 the quantities

$$
B^{\left(2 m d^{2}-1\right) e} b_{\rho \mu \nu}=B_{\rho \mu \nu}
$$


are symmetric polynomials, with integral coefficients, in the conjugates of $b_{0}$, of degree at most $\left(2 m d^{2}-1\right) d e$ in $b_{0}$. Hence, by Lemma 3.18 ,

$$
B_{\rho \mu \nu}^{*}=D_{0}^{\left(2 m d^{2}-1\right) d e} B_{\rho \mu \nu} \in \mathbf{Z} .
$$

Also, by Lemma 3.19,

$$
\begin{aligned}
\left|B_{\rho \mu \nu}^{*}\right| & \leqslant D_{0}^{\left(2 m d^{2}-1\right) d e}\left|B_{\rho \mu \nu}\right| \leqslant\left(D_{0}^{d} B\right)^{\left(2 m d^{2}-1\right) e}\left|b_{\rho \mu \nu}\right| \\
& \leqslant\left[(d+1)(2 h)^{d} / D_{0}^{d-2}\right]^{\left(2 m d^{2}-1\right) d e}\left|b_{\rho \mu \nu}\right| .
\end{aligned}
$$

From (3.15) we infer that

$$
\left|b_{\rho \mu \nu}\right|<\left(4 C_{1}\right)^{d}\left(2 R_{0}^{1 / e}\right)^{2 M e+d m} \leqslant\left(4 C_{1}\right)^{d}\left(2 R_{0}^{1 / e}\right)^{m d^{2}} .
$$

It follows that

$$
\left|B_{\rho \mu \nu}^{*}\right|<d^{4 d^{5}}(2 h)^{3 d^{6}}
$$

We now need Siegel's Lemma, whose proof is included for the sake of completeness.

3.24. Lemma. The system of equations

$$
\sum_{j=0}^{2 M} A_{i j} x_{j}=0, \quad\left|A_{i j}\right| \leqslant A, \quad A_{i j} \in \mathbf{Z}, \quad i=1,2, \ldots, M,
$$

has nontrivial integral solutions satisfying

$$
\left|x_{j}\right|<(2 M+1) A, \quad j=0,1, \ldots, 2 M .
$$

ProOF. Set

$$
y_{i}=\sum_{j=0}^{2 M} A_{i j} x_{j}, \quad i=1,2, \ldots, M,
$$

and let $x_{j}$ vary over the integers $0,1, \ldots, X$. Then $-N_{i} X \leqslant y_{i} \leqslant P_{i} X$ where

$$
-N_{i}=\sum_{\substack{j=0 \\ A_{i j}<0}}^{2 M} A_{i j}, \quad P_{i}=\sum_{\substack{j=0 \\ A_{i j}>0}}^{2 M} A_{i j}, \quad P_{i}+N_{i} \leqslant(2 M+1) A .
$$

Thus there are $(X+1)^{2 M+1}$ choices for the vector $\mathbf{x}=\left(x_{0}, x_{1}, \ldots, x_{2 M}\right)$ and no mere than

$$
((2 M+1) A X+1)^{M}<((2 M+1) A)^{M}(X+1)^{M}
$$

choices for the y-vectors. If

$$
(X+1)^{2 M+1} \geqslant((2 M+1) A)^{M}(X+1)^{M},
$$

which certainly holds if

$$
X+1 \geqslant(2 M+1) A,
$$

then two distinct $\mathbf{x}$-vectors, $\mathbf{x}_{1}$ and $\mathbf{x}_{2}$, give the same $\mathbf{y}$-vector and the vector $\mathbf{x}=\mathbf{x}_{1}-\mathbf{x}_{2}$ yields the desired solution. 
Using (3.23) we get the following

3.25. Corollary. There exist integers $C_{\rho} ; \rho=0,1, \ldots, 2 M$, not all zero, so that

and

$$
\sum_{\rho=0}^{2 M} C_{\rho} B_{\rho \mu \nu}^{*}=0, \quad 1 \leqslant \mu \leqslant \nu m / e, 1 \leqslant \nu<d,
$$

$$
\left|C_{\rho}\right|<m d^{2} \max \left|B_{\rho \mu \nu}^{*}\right|<d^{5 d^{5}}(2 h)^{3 d^{6}}
$$

For the $C_{\rho}$ in Corollary 3.25 we have

$$
\sum_{\rho=0}^{2 M} C_{\rho} F(x, y ; \rho)=Q(x, y)+O\left(x^{-1 / e}\right)
$$

where

$$
Q(x, y)=\sum_{\rho=0}^{2 M} C_{\rho} P(x, y ; \rho)
$$

is a polynomial, whose coefficients are symmetric polynomials with integral coefficients in the conjugates of $b_{n}, 0 \leqslant n \leqslant 2 M e+m<m d^{2}$.

Thus, as before, we have

$$
Q^{*}(x, y)=\left(D_{0}^{d} B\right)^{\left(2 m d^{2}-1\right) e} Q(x, y) \in \mathbf{Z}[x, y]
$$

and, by (3.15),

$$
\text { height } \begin{aligned}
Q^{*}< & \left((d+1)^{d}(2 h)^{d^{2}}\right)^{\left(2 m d^{2}-1\right) e}(2 M+1) d^{5 d^{5}}(2 h)^{2 d^{6}} \\
& \cdot\left(4 C_{1}\right)^{d}\left(2 R_{0}^{1 / e}\right)^{2 M e+m d}<d^{8 d^{5}}(3 h)^{5 d^{6}} .
\end{aligned}
$$

To sum up we have the following

\subsection{Lemma. The polynomial $Q^{*}$ satisfies}

$$
\operatorname{deg}_{y} Q^{*}<d, \quad \operatorname{deg}_{x} Q^{*} \leqslant 2 M+m d / e \leqslant d^{3} / e, \text { height } Q^{*}<d^{8 d^{5}}(3 h)^{5 d^{h}} \text {. }
$$

The error term in (3.26) consists of those terms $y^{\mu} / x^{\nu}$ in the sum on the left for which $\mu m-\nu e<0$. From (3.13) we infer, for $|x| \geqslant 2^{d} R_{0}>2^{e} R_{0}$, that

$$
|y|<2 C_{1}|x|^{m / e} \text {. }
$$

Hence (3.15) implies, for $|x| \geqslant 2^{d} R_{0}$, that the error terms, $E(x, y ; \rho)(\rho=$ $0,1, \ldots, 2 M)$, which are $O\left(x^{-1 / e}\right)$, in $F(x, y ; \rho)$, satisfy

$$
\begin{aligned}
|E(x, y ; \rho)| & <4 C_{1}^{d} \sum_{n=1}^{\infty}\left(2 R_{0}^{1 / e}\right)^{2 M e+d m+n}|x|^{-n / e}(d+1)\left(2 C_{1}\right)^{d} \\
& <4 d\left(8 C_{1}^{2}\right)^{d}\left(2 R_{0}^{1 / e}\right)^{m d^{2}+1}|x|^{-1 / e}
\end{aligned}
$$

Thus the error term, $E^{*}(x, y)$, in (3.26) satisfies

$$
\begin{aligned}
E^{*}(x, y) & \leqslant \sum_{\rho=0}^{2 M}\left|C_{\rho} \| E(x, y ; \rho)\right| \\
& <(2 M+1) d^{5 d^{5}}(2 h)^{3 d^{6}} \cdot 4 d\left(8 C_{1}^{2}\right)^{d}\left(2 R_{0}^{1 / e}\right)^{m d^{2}+1}|x|^{-1 / e}<1,
\end{aligned}
$$


provided

$$
|x| \geqslant(2 M+1)^{e} d^{5 d^{5} e}(2 h)^{3 d^{6} e}(4 d)^{e}\left(8 C_{1}^{2}\right)^{d e}\left(2^{e} R_{0}\right)^{m d^{2}+1} .
$$

This certainly holds when

$$
|x| \geqslant d^{5 d^{6}}(2 h)^{3 d^{7}} .
$$

Thus $Q^{*}(x, y)=0$ for all lattice points on (3.7) which satisfy (3.29). All $x$-coordinates of these lattice points therefore satisfy

$$
R(x)=\operatorname{res}_{y}\left(F(x, y), Q^{*}(x, y)\right)=0 .
$$

The resultant $R(x)$ is a determinant with no more than $d-1$ rows whose entries are coeffcients of powers of $y$ in $F$, and no more than $d$ rows whose entries are coefficients of powers of $y$ in $Q^{*}$. So, by using Hadamard's inequality and Lemma 3.27, we get, $h\left(Q^{*}\right)=$ height $Q^{*}$,

$$
\begin{aligned}
R(x) & \ll(2 d-1)^{d-1 / 2} h^{d-1} h\left(Q^{*}\right)^{d}\left(1+x+\cdots+x^{d}\right)^{d-1}\left(1+x+\cdots+x^{d^{3}}\right)^{d} \\
& \ll(2 d)^{d} h^{d-1} d^{8 d^{6}}(3 h)^{5 d^{7}}\left(1+x+\cdots+x^{d}\right)^{d-1}\left(1+x+\cdots+x^{d^{3}}\right)^{d}
\end{aligned}
$$

Hence

$$
\text { height } R<(2 d)^{d} h^{d-1} d^{8 d^{6}}(3 h)^{5 d^{7}}(d+1)^{d-1}\left(d^{3}+1\right)^{d}<d^{9 d^{6}}(3 h)^{6 d^{7}} .
$$

Therefore any zero of $R(x)$ satsisfies

$$
|x|<d^{9 d^{6}}(3 h)^{6 d^{7}} \text {. }
$$

The same bound works for $|y|$ from (3.28), since for $e<m \leqslant d$ the bound (3.30) could be reduced substantially. Summing up we have the following theorem.

3.31. TheOREM. Under Runge's Condition and Assumption 3.17, the solutions of the Diophantine equation (3.1) satisfy

$$
\max \{|x|,|y|\} \leqslant d^{9 d^{6}}(3 h)^{6 d^{7}} .
$$

4. The case $H_{z}\left(0, b_{0}\right)=0$. Here the successive equations for the Taylor coefficients $b_{n}$, which result from the equation $H(t, z)=0$, are not necessarily linear for all $n$; but they do become linear for all $n>n_{0}$. We develop the idea for general algebraic functions, following a method employed by Heine [2] (see Pólya and Szegö [12, Volume II, Problem VIII, §3]).

4.1. Lemma. Let $H(t, z)$ be an irreducible polynomial in $\mathbf{C}[t, z]$ with $\operatorname{deg}_{t} H \leqslant d_{0}$ and $\operatorname{deg}_{z} H=d_{2}$. Let

$$
D(t)=\operatorname{res}_{z}\left(H, H_{z}\right)
$$

be the resultant of $H$ and $H_{z}$. Then

$$
\operatorname{deg} D(t) \leqslant d_{0}\left(2 d_{2}-1\right) .
$$

Hence, if $n_{1}$ is the order of the zero of $D(t)$ at $t=0$ then

$$
n_{1} \leqslant d_{0}\left(2 d_{2}-1\right) \text {. }
$$


Proof. The polynomial $D(t)$ is the value of a determinant of size no greater than $\left(2 d_{2}-1\right) \times\left(2 d_{2}-1\right)$, whose entries are polynomials in $t$ of degree no greater than $d_{0}$.

4.2. Lemma. Let $z_{1}, z_{2}, \ldots, z_{d}$ be the solutions of $H(t, z)=0$, analytic in $a$ punctured neighborhood, $0<|t|<\delta$, of 0 . Let $n_{0}$ be the least integer so that

$$
\lim _{t \rightarrow 0} t^{-n_{0}-1}\left(z_{i}-z_{j}\right)=\infty \text { for } 1 \leqslant i<j \leqslant d_{2} .
$$

Then $n_{0} \leqslant n_{1} / 2 \leqslant d_{0}\left(d_{2}-\frac{1}{2}\right)$.

Proof. Write $H(t, z)=A_{0}(t)+A_{1}(t) z+\cdots+A_{d_{2}}(t) z^{d_{2}}$. Then

$$
D(t)=(-1)^{d_{2}\left(d_{2}-1\right) / 2} A_{d_{2}}(t)^{2 d_{2}-1} \prod_{1 \leqslant i<j \leqslant d_{2}}\left(z_{i}-z_{j}\right)^{2} .
$$

If the lemma were false then one of the factors, $\left(z_{i}-z_{j}\right)^{2}$, would have a zero of order at least $n_{1}+1$ at $t=0$. While some of the conjugates $z_{k}$ may have poles at $t=0$, these poles are cancelled by the zeros of $A_{d_{2}}(t)$. Thus it would follow that $D(t)$ has a zero of order greater than $n_{1}$ at $t=0$, in contradiction to Lemma 4.1.

4.3. Lemma. Let $H(t, z)$ be as in Lemma 4.1 and $n_{0}$ as in Lemma 4.2. Let $z=\sum_{n=0}^{\infty} b_{n} t^{n}$ be a formal power series solution of $H(t, z)=0$ which is therefore analytic in a neighborhood of 0 . Then the successive equations for $b_{n}$ in terms of $b_{0}, b_{1}, \ldots, b_{n-1}$ are linear of the form $B b_{n}+C_{n}=0$, provided $n>n_{0}$. Here $B$ is a polynomial in $b_{0}, b_{1}, \ldots, b_{n_{0}}$ and $C_{n}$ is a polynomial in $b_{0}, b_{1}, \ldots, b_{n-1}$.

Proof. Set

$$
z=\sum_{n=0}^{n_{0}} b_{n} t^{n}+t^{n_{0}+1} w
$$

and write

$$
H(t, z)=t^{M_{1}} K(t, w)
$$

where $M_{1}$ is chosen so that $K(t, w)$ is a polynomial in $t$ and $w$ and $K(0, w) \neq 0$. Then by Lemma 4.2 the solutions $w=w_{1}, w_{2}, \ldots, w_{d_{2}}$ of $K(t, w)=0$ have the property that only $w_{1}$ is bounded as $t \rightarrow 0$ while $w_{2}, w_{3}, \ldots, w_{d_{2}}$ tend to $\infty$. Hence, if we write

$$
K(t, w)=K_{0}(t)+K_{1}(t) w+\cdots+K_{d_{2}}(t) w^{d_{2}},
$$

then, expressing $K_{\delta}$ as elementary symmetric functions of $w_{1}, w_{2}, \ldots, w_{d_{2}}$, we get $K_{2}(0)=K_{3}(0)=\cdots=K_{d_{2}}(0)=0$ while $B=K_{1}(0) \neq 0$. Then coefficients of the $K_{\delta}$ are polynomials in $b_{0}, b_{1}, \ldots, b_{n_{0}}$. If we now set $w=\sum_{n=n_{0}+1}^{\infty} b_{n} t^{n}$ and collect coefficients of $t^{n}$ in (4.4) then we get an equation $B b_{n}+C_{n}=0$, where the terms collected in $C_{n}$ involve only $b_{0}, b_{1}, \ldots, b_{n-1}$.

We summarize the contents of Lemmas 4.1-4.3, and Lemma 3.21, whose proof is also valid in the present setting $H(t, z) \in \mathbf{C}[t, z]$, in the form of a quantitative version of a classical result. 
4.5. Theorem. Let $H(t, z)$ be an irreducible polynomial in $\mathbf{C}[t, z]$ with $\operatorname{deg}_{t} H(t, z)$ $\leqslant d_{0}$ and $\operatorname{deg}_{z} H(t, z)=d_{2}$. Let

$$
z=\sum_{n=0}^{\infty} b_{n} t^{n}
$$

satisfy $H(t, z)=0$ in a neighborhood of zero. Then there is a value of the index $n=n_{0}$ for which all the coefficients $b_{n}$ lie in the field generated by $b_{0}, b_{1}, \ldots, b_{n_{0}}$ and the coefficients of $H$. For $n>n_{0}$, the equation for $b_{n}$, obtained by substituting the power series expansion of $z$ into $H(t, z)=0$, is linear, of the form $B b_{n}+C_{n}=0$, where $B \neq 0$ is a polynomial in $b_{0}, b_{1}, \ldots, b_{n_{0}}$ and $C_{n}$ is a polynomial in $b_{0}, b_{1}, \ldots, b_{n-1}$. The coefficients of $B$ and $C_{n}$ are in the field of the coefficients of $H(t, z)$. A sufficient condition for $n_{0}=0$ is that $H_{z}\left(0, b_{0}\right) \neq 0$. In any case,

$$
n_{0} \leqslant d_{0}\left(d_{2}-\frac{1}{2}\right) \text {. }
$$

This last inequality has computational applications in the theory of algebraic functions (Hilliker [5]).

We now return to the Diophantine equation under consideration. Here $H(t, z) \in$ $\mathbf{Z}[t, z]$ and $d_{0} \leqslant d(e+m) \leqslant d(2 d-1), d_{2} \leqslant d$. Thus the constants $n_{0}, n_{1}$ satisfy

$$
n_{1} \leqslant d(2 d-1)(e+m) \leqslant d(2 d-1)^{2}, \quad n_{0} \leqslant n_{1} / 2 .
$$

In order to complete our estimations we need an algebraic integer $D^{*}$ so that $D^{*} b_{n}$ is an algebraic integer for $n=0,1, \ldots, n_{0}$. Then $\left(D^{*}\right)^{d_{\kappa}}$ has algebraically integral coefficients.

We have, according to (3.11),

$$
K_{1}(t)=t^{-M_{1}}\left[A_{1}(t)+2 A_{2}(t) \sum_{n=0}^{n_{0}} b_{n} t^{n}+\cdots+d_{2} A_{d_{2}}(t)\left(\sum_{n=0}^{n_{0}} b_{n} t^{n}\right)^{d_{2}-1}\right]
$$

The sum of the absolute values of the coefficients in each $A_{i}$ is no larger than $(d+1) h$ and, by (3.12),

$$
\sum_{n=0}^{n_{0}} \sqrt{b_{n}}<C_{1} \sum_{n=0}^{n_{0}} R_{1}^{n}<2 C_{1} R_{1}^{n_{0}}
$$

Thus (4.6) implies

$$
\begin{aligned}
\widehat{K_{1}(0)} & <(d+1) h \sum_{\delta=1}^{d-1}\left(2 C_{1} R_{1}^{\left.n_{0}\right)}\right)^{\delta} \\
& <2(d+1) h\left(2 C_{1} R_{1}^{n_{0}}\right)^{d-1} \\
& =2(d+1) h\left(2 C_{1}\right)^{d-1} R_{0}^{n_{0}(d-1) / e} .
\end{aligned}
$$

Now

$$
\begin{aligned}
n_{0}(d-1) / e & \leqslant d\left(d-\frac{1}{2}\right)(d-1)(1+m / e) \leqslant d\left(d-\frac{1}{2}\right)(d-1)(d+1) \\
& <d^{4}-d^{2} .
\end{aligned}
$$

Hence (3.12) and (3.6) give

$$
K_{1}(0) \mid<2(d+1) h\left(4(h+1) R_{0}^{2 d^{2} / e}\right)^{d-1} R_{0}^{d^{4}-d^{2}}<\left(4 d^{4} h^{2}\right)^{2 d^{5}}
$$


Now, in a manner precisely analogous to the way in which we proved Lemma 3.21 , we prove the following

4.7. Lemma. For each $n>0$, the number $B_{1}^{2 n-1} b_{n_{0}+n}$ is an algebraic integer, where

$$
B_{1}=\left(D^{*}\right)^{d} K_{1}(0) \text {. }
$$

The estimation of the "denominators" is therefore complete once we estimate $D^{*}$. For this purpose we estimate algebraic integers $D_{n}$ so that $D_{n} b_{i}$ is an algebraic integer for $i=0,1, \ldots, n ; n \leqslant n_{0}$.

Assume that we have already computed $D_{n}$. Then write $z=\left(b_{0}+b_{1} t\right.$ $\left.+\cdots+b_{n} t^{n}\right)+t^{n+1} z_{n}$ and construct the polynomial

$$
L_{n}\left(t, z_{n}\right)=t^{-l_{n}} H(t, z)
$$

where $l_{n}$ is chosen so that $L_{n}$ is a polynomial in $t$ and $z_{n}$ and $L_{n}\left(0, z_{n}\right) \neq 0$. The coefficients of $L_{n}\left(0, z_{n}\right)$ are polynomials, with integral coefficients, of degree at most $d$ in $b_{0}, b_{1}, \ldots, b_{n}$ and hence

$$
D_{n}^{d} L_{n}\left(0, b_{n+1}\right)=0
$$

is an equation with integral coefficients for $b_{n+1}$. We, thus, can choose $D_{n+1}$ as the leading coefficient in (4.8). As before we get

$$
\mid \overrightarrow{D_{n+1}}<(d+1) h{\overline{D_{n}}}^{d} \sum_{\delta=\mu}^{d}\left(\begin{array}{l}
\delta \\
\mu
\end{array}\right)\left(2 C_{1} R_{1}^{n}\right)^{\delta-\mu}
$$

where $\mu, 1 \leqslant \mu \leqslant d$, is the degree of (4.8) in $b_{n+1}$. Therefore

$$
D_{n+1}<\bar{D}_{n}^{d}(d+1) h \cdot 2^{d}\left(2 C_{1} R_{0}^{n / e}\right)^{d-1}<C_{2}\left(R_{0}^{n / e} \mid D_{n}\right)^{d},
$$

where

$$
C_{2}=(d+1) h 2^{d}\left(2 C_{1}\right)^{d-1}<\left(4 d^{2} h\right)^{4 d^{4} / e}
$$

Hence

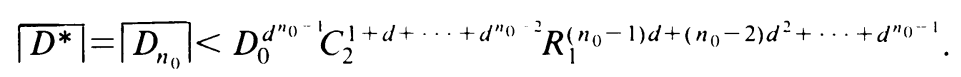

Now

$$
\left(n_{0}-1\right) d+\left(n_{0}-2\right) d^{2}+\cdots+d^{n_{0}-1}=\frac{d^{n_{0}+1}-1}{(d-1)^{2}}-\frac{n_{0}}{d-1}<2 d^{n_{0}}
$$

and therefore

$$
D^{*} \mid<\left(C_{2} R_{0}^{1 / e} D_{0}\right)^{2 d^{n_{0}}}<(4 d h)^{d^{2 d^{3}}} .
$$

The rest of the argument is exactly as before. We omit details.

4.9. THEOREM. If $F(x, y)$ satisfies Runge's Condition then the integral solutions $x, y$ of $F(x, y)=0$ satisfy

$$
\max \{|x|,|y|\}<(8 d h)^{d^{2 d^{3}}}
$$




\section{REFERENCES}

1. William John Ellison, Variations sur un thème de Carl Runge, Séminaire Delange-Pisot-Poitou (13e année, 1971/72), Théorie des Nombres, Fasc. 1, Exp. No. 9, Secrétariat Mathématique, Paris, 1973. MR 54 \#7369.

2. E. Heine, Handbuch der Kugelfunctionen. Theorie und Anwendungen. Vols. I, II, 2nd ed., G. Reimer, Berlin, 1878; 1881. See Jbuch. 10, 332; 13, 390-391.

3. David Lee Hilliker, An algorithm for solving a certain class of Diophantine equations. I, Math. Comp. 38 (1982), 611-626.

4. An algorithm for solving a certain class of Diophantine equations. II (to be submitted).

5. An algorithm for computing the values of the ramification index in the Puiseux series expansions of an algebraic function (to be submitted).

6. David Lee Hilliker and E. G. Straus, On Puiseux series whose curves pass through an infinity of algebraic lattice points, Bull. Amer. Math. Soc. (N.S.) 8 (1983), 59-62.

7. William Judson LeVeque, Topics in number theory, Vols. I, II, Addison-Wesley, Reading, Mass., 1956.

8. Edmond Maillet, Sur les équations indéterminées à deux et trois variables qui n'ont qu' un nombre fini de solutions en nombres entiers, J. Math. Pures Appl. 6 (1900), 261-277. An abstract appeared in C. R. Acad. Sci. Paris 128 (1899), 1383-1395. See Jbuch. 30, 188-189; 31, 190-191.

9. Sur une catégorie de'équations indéterminées n'ayant en nombres entiers qu' un nombre fini de solutions, Nouv. Ann. Math. 18 (1918), 281-292. See Jbuch. 46, 210.

10. Louis Joel Mordell, Diophantine equations, Academic Press, London and New York, 1969.

11. Harry Pollard and Harold G. Diamond, The theory of algebraic numbers, 2nd ed., Carus Mathematical Monographs, Number 9, Mathematical Association of America, Washington, D. C., 1975. This is a new edition of the work of Pollard of 1950.

12. G. Pólya and G. Szegö, Problems and theorems in analysis, Vols. I, II, Revised and enlarged transl. of 4th German ed., Die Grundlehren der Math. Wissenschaften, Bands 193, 216, Springer-Verlag, New York and Berlin, 1972, 1976; 1st German ed., Aufgaben und Lehrsätze aus der Analysis, Julius Springer, Berlin, 1925; 4th German ed., 1970, 1971; 1st German ed. also published in two volumes by Dover, New York, 1945.

13. C. Runge, Über ganzzahlige Lösungen von Gleichungen zwischen zwei Veränderlichen, J. Reine Angew. Math. 100 (1887), 425-435. See Jbuch. 19, 76-77.

14. A. Schinzel, An improvement of Runge's theorem on Diophantine equations, Comment. Pontificia Acad. Sci. 2 (1969), 1-9. MR 43 \# 1922.

15. Carl Ludwig Siegel, Approximation algebraischer Zahlen, Math. Z. 10 (1921), 173-213; Also in Gesammelte Abhandlungen, Vol. I, Springer-Verlag, Berlin and New York, 1966, pp. 6-46. See Jbuch. 48, $163-164$.

16. , Über einige Anwendungen diophantischer Approximationen, Abh. Preuss. Akad. Wiss. Phys. Math. Natur. Kl. 1 (1929). Also in Gesammelte Abhandlungen Vol. I, Springer-Verlag, Berlin and New York, 1966, pp. 209-266. See Jbuch. 56, 180-184.

17. Th. Skolem, Über ganzzahlige Löhungen einer Klasse unbestimmter Gleichungen, Norsk mat. Foren. Akrifter, Ser. I 10 (1922). See Jbuch. 48, 139.

18. Diophantische Gleichungen, Verlag von Julius Springer, Berlin, 1938, reprinted by Chelsea, New York, 1950.

\section{Department of Mathematics, University of California, Los Angeles, California 90024}

Current address (D. L. Hilliker): Department of Computer Science, California State University, Dominguez Hills, Carson, California 90747 